\title{
Évolution des polyamines dans les bourgeons et les rameaux de Pseudotsuga menziesii (Mirb) Franco à la suite du passage de l'état végétatif à l'état floral
}

\author{
EH Daoudi 1, M Bonnet-Masimbert 1, J Martin-Tanguy 2 \\ 1 INRA, Station d'amélioration des arbres forestiers, centre de recherche d'Orléans, \\ Ardon, 45160 Olivet; \\ 2 INRA, Station de physiopathologie végétale, BV 1540, 21034 Dijon Cedex, France
}

(Reçu le 28 février 1991; accepté le 23 mai 1991)

\begin{abstract}
Résumé - Les polyamines de sapin de Douglas (Pseudotsuga menziesii) ont été analysées et quantifiées par chromatographie liquide haute performance (CLHP) après extraction des tissus et dansylation. La détection est fluorimétrique. L'étude a porté sur des bourgeons morphologiquement distincts d'un clone (1101) et sur des rameaux porteurs de bourgeons sexués et/ou végétatifs provenant de deux clones (1101 et 1200). La distribution des polyamines (putrescine, spermidine, et spermine) est différente suivant que l'organe est végétatif ou floral. Par comparaison avec les bourgeons sexués, les bourgeons végétatifs sont caractérisés par une plus forte teneur en putrescine qui contraste avec une moindre teneur en spermidine. Une forte teneur en spermidine caractérise surtout les bourgeons mâles. Par ailleurs une augmentation générale de la teneur de ces polyamines est observée dans les rameaux (tige, aiguilles, bourgeons) lorsque ceux-ci portent des bourgeons sexués en plus des bourgeons végétatifs. D'une façon générale, la spermidine est plus abondante dans les bourgeons que dans les rameaux. De plus la spermine n'a été trouvée que dans les bourgeons sexués. Enfin, le rapport entre la putrescine et la spermidine permet de caractériser le passage des bourgeons de l'état végétatif à l'état floral, que l'on s'intéresse aux bourgeons eux-mêmes ou aux rameaux qui les portent.
\end{abstract}

sapin de Douglas / Pseudotsuga menziesii / bourgeon / conifère / rameau / polyamine / floraison

Summary - Polyamine evolution in buds and shoots of Douglas fir (Pseudotsuga menziesii) after the transition from vegetative development to sexual development. After methanolic extraction and dansylation, polyamines (putrescina, spermidine, spermine) of shoots and buds of Douglas fir (Pseudotsuga menziesii) were separated using reverse phase high performance liquid chromatography (HPLC). They were quantified by fluorescence detection (fig 1). On plant material collected in fall, free polyamine levels were measured in morphologically distinct vegetative, male and female buds of ramets of one clone (1101) and in shoots (needles, stem, and buds) bearing only vegetative buds or vegetative as well as male and female buds of ramets of two clones (1101 and 1200). The distribution of polyamines was different between sexual and vegetative buds. Putrescine was the dominant polyamine in vegetative buds, while spermidine predominated in floral buds (fig 3). The highest concentration of spermidine was observed in male buds. Furthermore, spermine was only found in the sexual buds (fig 3). All polyamines also increased in the shoots bearing sexual buds (fig 4) compared to shoots with only vegetative buds. In general, the spermidine level was higher in buds than in shoots. Finally, the ratio between putrescine and spermidine in sexual buds as well as in shoots bearing these kinds of buds was much lower than in vegetative organs (tables I and II). The possibility of using these polyamine contents as physiological markers of sexual differentiation is discussed. 


\section{INTRODUCTION}

Les polyamines telles que la putrescine (PUT), la spermidine (SPD) et la spermine (SPM) constituent un ensemble de substances naturelles qui jouent probablement un rôle important dans la régulation de la croissance et du développement des végétaux (Martin-Tanguy et al, 1984; Galston et Kaur-Sawhney, 1990). Les polyamines semblent intervenir dans plusieurs processus physiologiques, tels que : action inhibitrice de la synthèse de l'éthylène (Apelbaum et al, 1981; Suttle, 1981), interaction avec les acides nucléiques (Bagni et al, 1981) et action anti-senescence (Altman, 1982; Muhitch et al, 1983; Galston et Kaur-Sawhney, 1987a). Des accumulations de putrescine ont été détectées dans des conditions physiologiques très diverses : en cas de déficience minérale (Smith, 1970; Basso et Smith, 1974), d'alimentation riche en azote ammoniacal (Le Rudulier, 1978), en réponse à un choc osmotique (Flores et Galston, 1982) ou à une diminution $\mathrm{du} \mathrm{pH}$ du milieu nutritif (Flores et Galston, 1984; Tiburcio et al, 1986). Plus particulièrement, la présence de certaines polyamines chez le tabac semble être liée à l'état floral, et cela juste après l'induction florale (Cabanne et al, 1977, 1981). Tiburcio et al (1988) ont trouvé que la différenciation des bourgeons végétatifs en bourgeons floraux chez le tabac s'accompagne d'une augmentation de la teneur en putrescine mais surtout en spermidine. De plus, par des applications exogènes, Rohozinski et al (1986) ont montré qu'une infiltration de polyamines (PUT, SPD ou SPM) provoquait une augmentation de la floraison chez le pommier.

Le présent travail concerne l'étude qualitative et quantitative du contenu en polyamines des bourgeons mâles, femelles et végétatifs du sapin de Douglas (Pseudotsuga menziesii) d'une part, et des ra- meaux portant ces bourgeons d'autre part. II s'agit en particulier de savoir si les variations observées au niveau des rameaux peuvent constituer des marqueurs biochimiques du processus de sexualisation (Bonnet-Masimbert, 1989). À partir de là, notre objectif sera à terme de reconnaître précocement, avant que les bourgeons ne soient morphologiquement distincts, l'évolution vers l'état végétatif ou floral, des méristèmes portés par un rameau.

\section{MATÉRIEL ET MÉTHODES}

\section{Matériel végétal}

Des rameaux de sapin de Douglas ont été prélevés le 15 décembre 1987. Ces prélèvements sont faits à partir de 6 plants greffés, âgés de 8 ans depuis la greffe, issus de deux clones (1101 et 1200 ), et élevés en conteneurs en pépinière à I'Institut National de la Recherche Agronomique à Orléans. Les rameaux prélevés proviennent tous de pousses formées en 1987 sur les rameaux du verticille formé en 1985. Deux types de prélèvements ont été effectués : 1) 63 bourgeons mâles $(2,380 \mathrm{~g}$ de matière fraîche (MF)), 17 femelles $(1,663 \mathrm{~g}$ de MF), et 16 végétatifs $(1,015 \mathrm{~g}$ de MF) prélevés séparément sur les plants du clone $1101 ; 2$ ) trois rameaux complets (tige, aiguilles, bourgeons) individualisés, prélevés sur les mêmes arbres et portant selon les cas des bourgeons végétatifs seuls, ou associés à des bourgeons mâles et femelles. Ces rameaux sont prélevés sur les 2 clones 1101 et 1200. Les rameaux végétatifs du clone 1101 (4,87 $\mathrm{g}$ de MF) portaient en moyenne trois bourgeons végétatifs alors que ceux du clone 1200 $(2,70 \mathrm{~g}$ de MF) en portaient quatre. Les rameaux sexués du clone 1101 (4,28 g de MF) portaient en moyenne 4 bourgeons végétatifs, 11 mâles et 3 femelles tandis que ceux du clone 1200 (2,87 g de MF) portaient en moyenne 3 bourgeons végétatifs, 7 mâles et 2,5 femelles. Après la récolte, chaque échantillon est pesé (poids exprimé en MF) et placé dans une solution d'acide chlorydrique $(\mathrm{HCl}+\mathrm{N})\left(20 \mathrm{ml} \cdot \mathrm{g}^{-1}\right.$ $\mathrm{MF}$ ), puis conservé à $-5^{\circ} \mathrm{C}$ en chambre froide. 


\section{Méthodes}

\section{Extraction}

L'extraction des polyamines, réalisée de facon identique pour les clones 1101 et 1200 , consiste à broyer le matériel végétal à l'aide d'un polytron en ajoutant à la solution $\mathrm{HCl} 1 \mathrm{~N}$ du méthanol (30 ml. $\mathrm{g}^{-1} \mathrm{MF}$ ) contenant $0.1 \%$ de $\mathrm{HCl} 1 \mathrm{~N}$ et $0.1 \%$ de bisulfite de sodium utilisé comme antioxydant. Á cette solution d'extraction est ajoutée $0.1 \mu \mathrm{mol} \cdot \mathrm{g}^{-1} \mathrm{MF}$ de 1,6-diaminohexane (HDA) comme témoin interne pour l'estimation du rendement de la purification et de la séparation (Smith et Davies, 1987). L'extrait est filtré sur verre fritté $\left(n^{\circ} 3\right)$ et le résidu lavé 3 fois avec $15 \mathrm{ml} \mathrm{HCl}$ \ $\mathrm{N}$. Le filtrat est séché sous vide à l'évaporateur rotatif à $40^{\circ} \mathrm{C}$, repris dans $15 \mathrm{ml}$ $\mathrm{HCL}+\mathrm{N}$, puis filtré sur verre fritté $\left(n^{\circ} 2\right)$. Ce filtrat est alors traité dans l'ampoule à décanter par $15 \mathrm{ml}$ d'eau et $30 \mathrm{ml}$ d'acétate d'éthyle pour éliminer les lipides et les composés phénoliques. La phase aqueuse est concentrée à sec à $40^{\circ} \mathrm{C}$ à l'aide d'un évaporateur rotatif. L'extrait est ensuite repris par $\mathrm{HCl} 1 \mathrm{~N}$ à raison de $1 \mathrm{ml} \cdot \mathrm{g}^{-1} \mathrm{MF}$.

\section{Dansylation des polyamines}

Le protocole employé pour la formation des dérivés dansylés d'amines est adapté de la méthode décrite par Seiler et Wiechman (1970). Une fraction aliquote $(200 \mu l)$ de l'échantillon ou du témoin est saturée avec $100 \mathrm{mg}$ de carbonate de sodium. On traite ensuite cette fraction par $600 \mu \mathrm{l}$ d'une solution de chlorure de dansyle (1-diméthylamino-naphtalène-5-sulfonyl chloride) dans l'acétone $\left(10 \mathrm{mg} \cdot \mathrm{ml}^{-1}\right)$. Le mélange est laissé à incuber pendant $16 \mathrm{~h}$ à l'obscurité à 20 ${ }^{\circ} \mathrm{C}$. Le chlorure de dansyle en excès est ensuite éliminé par addition de $300 \mu \mathrm{l}$ d'une solution aqueuse de proline à $150 \mathrm{mg} \cdot \mathrm{ml}^{-1}$. L'extrait est remis pendant 30 min à l'obscurité. Les polyamines dansylées sont extraites par $1 \mathrm{ml}$ d'acétate d'éthyle. Après agitation des tubes pendant $1 \mathrm{~min}$, la phase supérieure d'acétate d'éthyle contenant les polyamines est évaporée à sec sous azote, puis le résidu redissout dans $1 \mathrm{ml}$ de méthanol absolu, est conservé à $-20^{\circ} \mathrm{C}$.

\section{Analyse des polyamines par CLHP}

Les polyamines sont analysées et quantifiées par chromatographie liquide haute performance (CLHP). Pour le clone 1101 on a utilisé un appareil CLHP (Waters assoc). La détection est réalisée par fluorimétrie (Perkin Elmer, Model 650$10 \mathrm{LC}$, volume de cellule $8 \mu \mathrm{l}$ ) (excitation à 340 $\mathrm{nm}$; émission à $455 \mathrm{~nm}$ ). Pour le clone 1200, la séparation des polyamines a été réalisée sur un appareil CLHP (Merck LC41B). La détection est faite par fluorimétrie (Gilson, Model 121, volume de cellule $9 \mu \mathrm{l}$ ) (excitation à $340 \mathrm{~nm}$; émission à 405-650 nm). Les polyamines après dansylation sont séparées sur une colonne $(4,6 \mathrm{~mm} x$ $250 \mathrm{~mm}$ ) Ultrasphère $C_{18}$ (silice greffée d'octadécylsilane) (ODS; dp (diamètre des particules) $5 \mu \mathrm{m}$ ) en phase inverse par un gradient binaire méthanol-eau. Le pourcentage de méthanol augmente linéairement de 60 à $95 \%$ en $23 \mathrm{~min}$, puis atteint $100 \%$ en $2 \mathrm{~min}$, demeure fixe $5 \mathrm{~min}$, et enfin retourne à $60 \%$ de méthanol en $10 \mathrm{~min}$. Le débit est de $1 \mathrm{ml} \cdot \mathrm{min}^{-1}$.

\section{Identification et quantification}

Un mélange de témoins de polyamines (PUT, HDA, SPD et SPM) à une concentration de $10^{-3} \mathrm{M}$ est dansylé dans les conditions précédemment décrites puis injecté en CLHP (fig 1). L'identité des temps de rétention des pics servira ultérieurement pour présumer de l'identité des polyamines extraites des échantillons végétaux. La quantification se fait par référence à des courbes étalons (fig 2) construites en injectant successivement des quantités connues de chacun des témoins et en déterminant à l'aide d'un intégrateur la surface correspondante des pics.

\section{Expression des résultats}

Pour estimer la précision des mesures, une série de trois extractions a été effectuée sur un même échantillon de matériel frais. Pour chaque extrait, trois dosages des polyamines ont été réalisés. Le coefficient de variation sur l'ensemble des neuf mesures ainsi obtenues est de 0,5\% pour PUT et $3 \%$ pour SPD. Par ailleurs, le ren- 


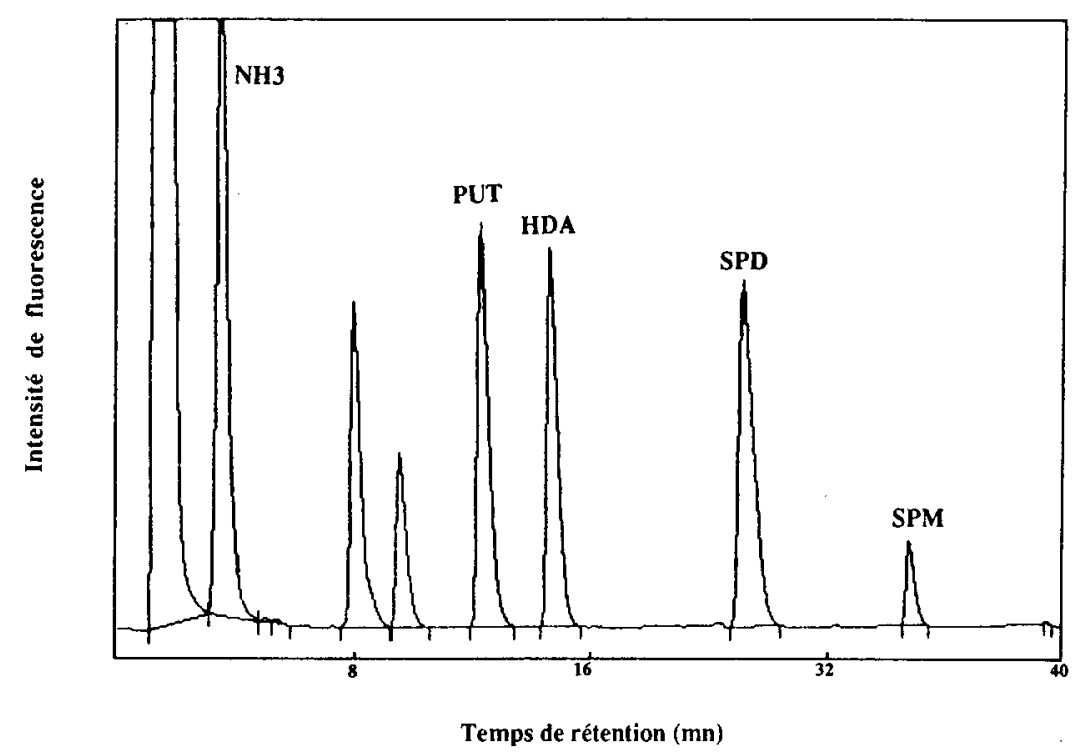

Fig 1. Chromatogramme d'un mélange de témoins dansylés constitué par $1 \mathrm{nmol}$ de putrescine (PUT), 1,6-diaminohexane (HDA), spermidine (SPD) et $0,11 \mathrm{nmol}$ de spermine (SPM).

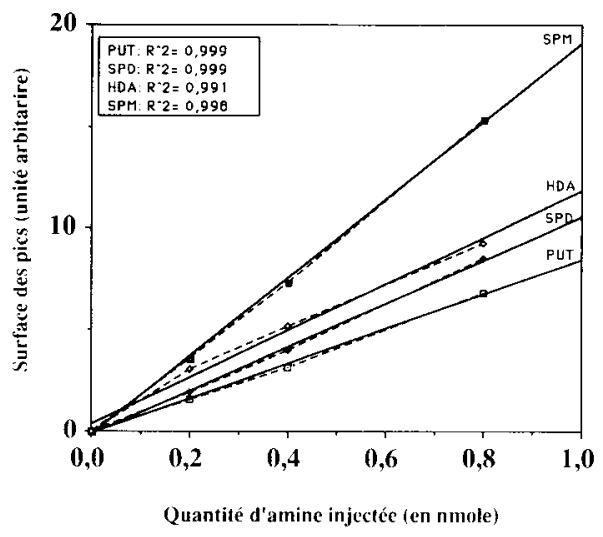

Fig 2. Régressions linéaires et coefficients de corrélation (R) des courbes étalons témoins représentant la relation entre la quantité injectée en nmol des polyamines dansylées (putrescine (PUT), spermidine (SPD), 1,6-diamino-hexane (HDA), et spermine (SPM)) et la surface des pics déterminée par un intégrateur. Traits pointillés : droites expérimentales et traits pleins : droites de régressions. dement de purification déterminé à l'aide du témoin HDA est en moyenne de $52 \%$.

\section{RÉSULTATS}

\section{Polyamines dans les bourgeons}

Dans nos conditions d'analyse, trois différentes polyamines (PUT, SPD et SPM) ont pu être détectées dans les bourgeons de Douglas (clone 1101). Le contenu de chaque type de bourgeons en chacune des polyamines est reporté sur la figure 3 . Ceci fait apparaître des différences à la fois qualitatives et quantitatives entre les différents types de bourgeons. La PUT est plus abondante dans les bourgeons végétatifs que dans les bourgeons sexués. Par contre, la SPD est plus abondante dans les bourgeons sexués et plus spéciale- 


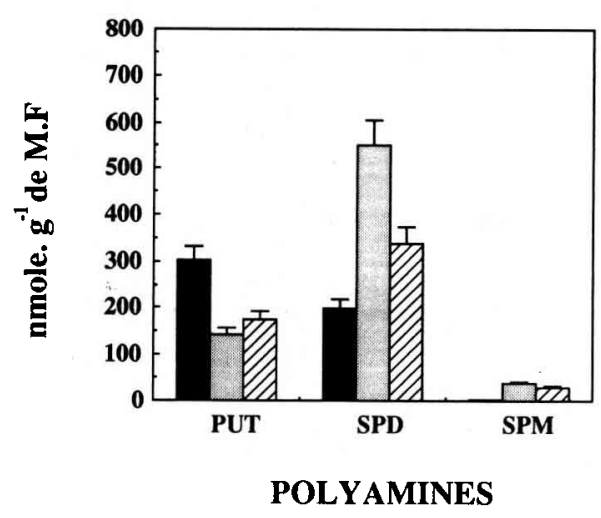

Fig 3. Teneur en putrescine (PUT), spermidine (SPD) et spermine (SPM) des bourgeons végétatifs $\square$, mâles $\square$ et femelles $\square$ (clone 1101). Les barres représentent les intervalles de confiance.

ment dans les bourgeons mâles. La SPM n'a été detectée que dans les bourgeons sexués et en quantité beaucoup plus faible que PUT et SPD. Le rapport PUT/SPD (tableau 1) distingue très clairement les bourgeons végétatifs des bourgeons sexués.

\section{Polyamines dans les rameaux}

La figure 4 met en évidence des variations quantitatives importantes aussi bien pour

Tableau I. Comparaison du rapport putrescine (PUT) sur spermidine (SPD) entre bourgeons végétatifs, mâles et femelles (clone 1101). * Coefficient de variation.

Végétatifs $\begin{gathered}\text { Bourgeons } \\ \text { Mâles } \quad \text { Femelles }\end{gathered}$

\section{PUT/}

SPD $1,50 \pm 0,250^{*} 0,26 \pm 0,043^{*} 0,52 \pm 0,086^{*}$
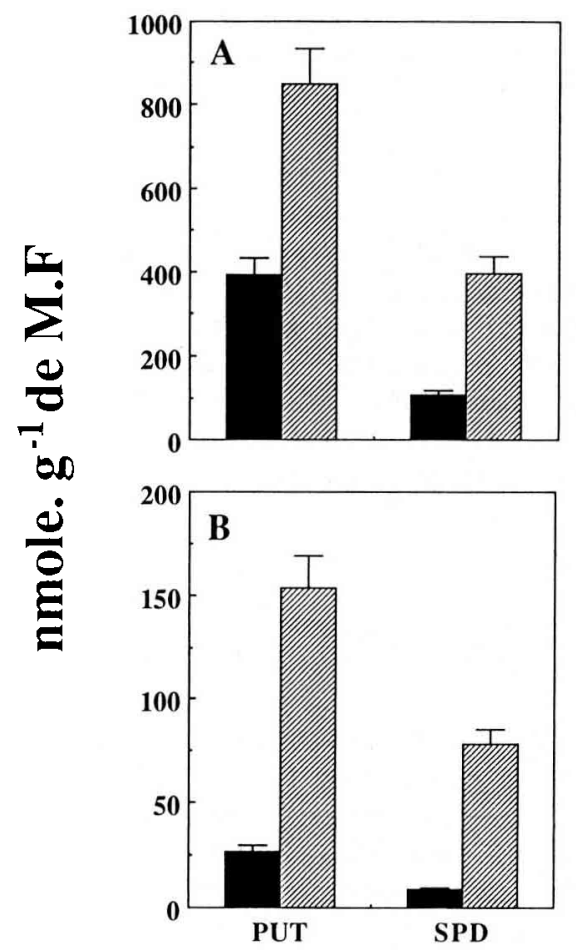

POLYAMINES

Fig 4. Teneur en putrescine (PUT) et spermidine (SPD) des rameaux : porteurs de bourgeons végétatifs $\square$ ou porteur de bourgeons végétatifs et sexués 1 . A : clone 1101 et $B$ : clone 1200. Les barres représentent les intervalles de confiance.

le clone 1101 que pour le clone 1200 entre les rameaux portant uniquement des bourgeons végétatifs et ceux qui portent à la fois des bourgeons mâles, femelles et végétatifs. On note que la PUT est 2,2 (clone 1101) à 5,7 (clone 1200) fois plus abondante dans les rameaux porteurs de bourgeons sexués que dans les rameaux exclusivement végétatifs. Quant à la SPD elle est 3,6 (clone 1101) à 8,7 (clone 1200) 
fois plus abondante dans les rameaux porteurs de bourgeons sexués que dans les rameaux porteurs de bourgeons végétatifs.

Par ailleurs, on note pour ces deux polyamines l'existence de différences quantitatives relativement importantes entre les clones 1101 et 1200 , ce qui suggère un effet clonal. Mais si l'on s'intéresse au rapport PUT/SPD, on constate qu'il est de même ordre de grandeur chez ces deux clones (tableau II), permettant ainsi de distinguer les rameaux strictement végétatifs des rameaux porteurs de bourgeons sexués.

\section{DISCUSSION ET CONCLUSION}

Si nous nous intéressons aux seuls bourgeons, on constate donc d'abord que chez le clone de sapin de Douglas que nous avons analysé, les bourgeons végétatifs sont caractérisés par une plus forte teneur en putrescine qui contraste avec une moindre teneur en spermidine. Une forte teneur en spermidine caractérise en particulier surtout les bourgeons mâles. Ceci rejoint l'observation faite par KaurSawhney et al (1988) qui ont montré chez le tabac cultivé in vitro à partir d'entre-

Tableau II. Comparaison du rapport PUT sur SPD entre rameaux végétatifs et rameaux sexués (clones 1101 et 1200). ${ }^{*}$ Coefficient de variation.

\begin{tabular}{ccc}
\hline$N^{\circ}$ Clone & $\begin{array}{c}\text { Rameaux } \\
\text { porteurs de } \\
\text { bourgeons } \\
\text { végétatifs }\end{array}$ & $\begin{array}{c}\text { Rameaux } \\
\text { porteurs de } \\
\text { bourgeons } \\
\text { végétatifs et sexués }\end{array}$ \\
\hline 1101 & $3,660 \pm 0,61^{*}$ & $2,140 \pm 0,356^{*}$ \\
1200 & $3,077 \pm 0,512^{*}$ & $1,973 \pm 0,382^{*}$ \\
\hline
\end{tabular}

nœuds de rameaux floraux immatures, que la formation des bourgeons végétatifs s'accompagnait d'une prédominance de la putrescine alors que celle des bourgeons floraux était liée à une prédominance de la spermidine. Par ailleurs, ces mêmes auteurs ont montré que l'addition de cyclohexylamine (inhibiteur de la spermidinesynthase) dans le milieu de culture, contenant une auxine et une cytokinine ayant une concentration de $1 \mu \mathrm{M}$ chacune, inhibe la différenciation des bourgeons floraux, alors qu'en son absence, 94\% de bourgeons floraux étaient formés. Cela montre le rôle de la spermidine dans la différenciation des bourgeons floraux. II apparaît que la transformation de la putrescine en spermidine est particulièrement importante dans le contrôle des divisions cellulaires, et que c'est la spermidine (et la spermine) qui est essentielle dans la transition de la phase $G \rightarrow S$ du cycle mitotique (Galston et Kaur-Sawhney, 1987b). Toutefois l'absence d'étude cinétique dans notre travail ne nous permet pas de dire si l'augmentation de spermidine constatée dans les bourgeons sexués, comme dans les rameaux qui les portent, se produit dès la phase d'initiation florale ou bien si, comme cela a été montré par Fiala et al (1988) sur le bulbe d'Iris hollandica, la remontée de la spermidine est d'abord précédée par une chute brutale.

D'autre part, la présence de spermine mérite d'être soulignée car cela semble vraiment caractéristique des bourgeons sexués. On notera au passage qu'on ne la retrouve pas dans les rameaux. II pourrait s'agir là d'un marqueur assez typique des bourgeons sexués. Tiburcio et al (1988) ont montré chez le tabac cultivé in vitro à partir d'explants d'entre-nœuds de rameaux floraux immatures, que la teneur en spermine est 5 fois plus élevée dans les bourgeons floraux que dans les bourgeons végétatifs. 
Dans le cas des rameaux, la figure 4 montre que pour les deux clones le contenu global en polyamines est sensiblement plus élevé lorsque ceux-ci portent des bourgeons sexués que lorsqu'ils sont strictement végétatifs. De même une augmentation des polyamines est observée à l'extrémité de la tige de tabac (un bourgeon terminal plus un fragment de tige de 1 à $2 \mathrm{~cm}$ portant cinq feuilles peu développées) au moment de l'induction florale (Perdrizet et Prévost, 1981).

Enfin, pour le clone 1101, pour lequel l'étude a porté à la fois sur les bourgeons pris séparément et sur les rameaux porteurs de bourgeons, et bien qu'il s'agisse d'échantillons différents prélevés sur les mêmes arbres, il semble qu'il y ait un certain équilibre, au moins pour la putrescine, entre rameaux et bourgeons lorsque ceuxci sont végétatifs. À l'inverse, lorsqu'il y a présence de bourgeons sexués, la teneur en putrescine des rameaux est sensiblement plus importante que celle des bourgeons. La spermidine, quant à elle, est systématiquement plus abondante (environ 2 fois) dans les bourgeons que dans les rameaux, que ceux-ci soient végétatifs ou sexués. Mais là encore, la sexualisation s'accompagne d'une augmentation de la teneur en spermidine. Donc, chez le Douglas, la quantité élevée de spermidine (et de spermine) dans les bourgeons rejoint les observations faites par d'autres auteurs qui ont confirmé que la biosynthèse et la concentration des polyamines sont souvent très élevées dans les tissus méristématiques. Par exemple, une telle augmentation de la teneur en spermidine et en spermine a été trouvée aussi bien dans les bourgeons de Picea abies (L) Karst (Königshofer, 1989) que dans la zone apicale des semis de Lens culinaris et Pisum sativum (Federico et Angelini, 1988).

Si l'objectif est la recherche d'un marqueur de l'état floral, le rapport putrescine sur spermidine (tableaux I et II) peut cons- tituer un premier élément. On constate en effet qu'il est le plus élevé lorsqu'il y a état végétatif, et ceci quel que soit le matériel végétal analysé. Bien entendu, dans la présente étude, les bourgeons sont morphologiquement distincts. Mais cette différence de composition en polyamines de rameaux provenant de mêmes arbres répondrait au souhait d'identifier au sein d'un même arbre les rameaux à vocation sexuée des rameaux à vocation strictement végétative. II resterait à vérifier à partir de quand, par rapport à la période d'initiation florale, cette distinction apparaît. Pour ce qui est des bourgeons, un résultat identique a été trouvé chez le tabac pour qui le rapport putrescine sur la somme de la spermidine et de la spermine est 2,3 fois plus élevé dans les bourgeons végétatifs que dans les bourgeons floraux (Tiburcio et al, 1988).

Enfin, on peut se demander si au delà d'un rôle de marqueur, les polyamines ne seraient pas susceptibles d'intervenir dans la sexualisation des bourgeons. L'effet positif de l'application exogène de putrescine, spermidine et spermine sur la floraison du pommier milite dans ce sens (Costa et Bagni, 1983; Rohozinski et al, 1986). Ceci est peut-être aussi à mettre en relation avec la forte augmentation d'arginine, précurseur de la synthèse des polyamines (Slocum et al, 1984), à la suite de fertilisation azotée ayant entraîné une floraison ultérieure, notamment chez Pinus elliotii (Barnes et Bengston, 1968), Pinus banksiana lam et Picea mariana lam (Mill) BSP (Kim et al, 1987), et aussi chez Picea glauca (Moench) Voss (Steward et Durzan, 1965). De même sur Pseudotsuga menziesii, Ebell et al (1970) ont montré qu'une application de nitrate au moment du débourrement végétatif multiplie par 2 à 7 la production de cônes femelles l'année suivante et qu'elle s'accompagne d'une accumulation d'acides aminés basiques, notamment l'arginine. 
Des résultats préliminaires (étude en cours) semblent indiquer que chez le sapin de Douglas une application des Gibbérellines (GA) $A 4$ et $A 7$, qui stimule l'initiation florale (Bonnet-Masimbert et Zaerr, 1987; Pharis et al, 1987), entraîne une augmentation de putrescine mais surtout de spermidine. Dai et al (1982) ont montré chez Pisum sativum que la croissance des entre-nœuds induite après un traitement par $\mathrm{GA}_{3}$ s'accompagnait d'une augmentation de l'activité de l'arginine décarboxylase (ADC) et de la teneur en polyamines. De même, l'injection de $\mathrm{GA}_{20}$ au niveau de l'entre-nœud, sous le bourgeon apical de Pisum sativum, entraîne une augmentation de la croissance des entre-nœuds, de la taille du bourgeon apical, et de la teneur en spermidine, mais pas de celle de la putrescine ou de la spermine (Smith et $\mathrm{Da}$ vies, 1985). Enfin, Smith et al (1985) ont suggéré que les polyamines ne jouaient pas de rôle dans l'élongation des cellules, mais qu'elles affectaient peut-être la prolifération cellulaire.

En conclusion, cette étude préliminaire des polyamines chez le sapin de Douglas révèle qu'une synthèse accrue des polyamines accompagne la sexualisation des bourgeons et se manifeste aussi dans les rameaux qui les portent. Cette différence de composition en polyamines de rameaux provenant des mêmes arbres semble particulièrement intéressante car elle ouvre la perspective de l'utilisation des polyamines comme marqueurs de la floraison. Resterait à vérifier à partir de quand cette distinction apparaît. Dans ce but, une étude cinétique de l'évolution des polyamines est en cours depuis le débourrement végétatif (mai) jusqu'à l'automne, ce qui recouvre la période pendant laquelle l'initiation florale prend place. Elle porte donc sur des rameaux dont les jeunes bourgeons en développement ne sont pas au départ morphologiquement distincts et appartenant à des plants soumis ou non à un traitement susceptible de provoquer la floraison.

\section{RÉFÉRENCES}

Altman A (1982) Retardation of radish leaf senescence by polyamines. Physiol Plant 54, 189-193

Apelbaum A, Burgoon AC, Anderson JD, Lieberman $M$ (1981) Biosynthesis of ethylene in higher plant tissue and fruit protoplasts. Plant Physiol 68, 453-456

Bagni N, Serafini-Fracassini D, Torrigiani $P$ (1981) Polyamines and growth in higher plants. In: Advances in polyamine research (CM Caldarera et al, eds) Raven Press, New York, Vol 3, 377-388

Basso LC, Smith TA (1974) Effect of mineral deficiency on amine formation in higher plants. Phytochemistry 13, 875-883

Barnes RL, Bengtson GW (1968) Effects of fertilization, irrigation and cover cropping on flow-ering and on nitrogen and soluble sugar composition of slash pine. For Sci 14, 172179

Bonnet-Masimbert M (1989) Promotion of flowering in conifers: from the simple application of a mixture of gibberellins to more integrated explanations. Ann Sci For 46 (suppl), 27s$33 \mathrm{~s}$

Bonnet-Masimbert M, Zaerr JB (1987) Hormonal control of tree growth. 2. The role of plant growth regulators in promotion of flowering. Plant Growth Regul 6, 13-35

Cabanne F, Martin-Tanguy J, Martin C (1977) Phénolamides associées à l'induction florale et à l'état reproducteur de Nicotiana tabacum var Xanthi nc. Physiol Vég 15, 429-443

Cabanne F, Dalebroux M, Martin-Tanguy J, Martin C (1981) Hydroxycinnamic acid amides and ripening to flower of Nicotiana tabacum var Xanthi nc. Physiol Plant 53, 399-404

Costa G, Bagni N (1983) Effects of polyamines on fruit-set of apple. Hortscience 18(1), 59-61 
Dai YR, Kaur-Sawhney R, Gaiston AW (1982) Promotion by gibberellic acid of polyamine biosynthesis in internodes of light-grown dwarf peas. Plant Physiol 69, 103-105

Ebell LF, Mc Mullan EE (1970) Nitrogenous substances associated with differential cone production responses of Douglas fir to ammonium and nitrate fertilization. Can $J$ Bot 48, 2169-2177

Federico R, Angelini R (1988) Distribution of polyamines and their related catabolic enzyme in etiolated and light-grown leguminosae seedlings. Planta 173, 317-321

Fiala V, Le Nard M, Querou Y, Jolivet E (1988) La spermidine, marqueur moléculaire de l'induction florale chez le bulbe d'Iris hollandica. CR Acad Sci Paris Sér III 306, 579-582

Flores HE, Galston AW (1982) Polyamines and plant stress: activation of putrescine biosynthesis by osmotic shock. Science 217, 12591261

Flores HE, Galston AW (1984) Osmotic stressinduced polyamine accumulation in cereal leaves. I. Physiological parameters of response. Plant Physiol 75, 102-109

Galston AW, Kaur-Sawhney R (1987a) Polyamines and senescence in plants. In: Plant senescence: its biochemistry and physiology (WW Thomson, EA Nothnagel, RC Huffaker, eds) The American Society of Plant Physiologists, 167-181

Galston AW, Kaur-Sawhney R (1987b) Polyamines as endogenous growth regulators. In: Plant hormones and their role in plant growth and development (PJ Davies, ed) Martinus Nijhoff Publ, Dordrecht, 280-295

Galston AW, Kaur-Sawhney R (1990) Polyamines in plant physiology. Plant Physiol 94, 406-410

Kaur-Sawhney R, Tiburcio AF, Galston AW (1988) Spermidine and flower-bud differentiation in thin-iayer explants of tobacco. Planta $173,282-284$

Kim YT, Glerum C, Stoddart J, Colombo SJ (1987) Effect of fertilization on free amino acid concentrations in black spruce and jack pine containerized seedlings. Can J For Res $17,27-30$
Königshofer $H$ (1989) Seasonal changes in polyamines content in different parts of juvenile spruce trees (Picea abies (L) Karst). Plant Physiol 134, 736-740

Le Rudulier D (1978) Utilisation de différentes formes d'azote et accumulation de putrescine par les plantules de Glycine max (L) Merr privées de cotylédons. Thèse, Rennes

Martin-Tanguy J, Margara J, Martin C (1984) Phénolamides et induction florale de Chichorium intybus dans différentes conditions de culture en serre ou in vitro. Physiol Plant 61, 259-262

Muhitch MM, Edwards LA, Fletcher JS (1983) Influence of diamines on the senescence of plant suspension cultures. Plant Cell Rep 2, 82-84

Perdrizet E, Prévost $J$ (1981) Aliphatic and aromatic amines during development of Nicotiana tabacum. Phytochemistry 20(9), 21312134

Pharis RP, Webber JB, Ross SD (1987) The promotion of flowering by gibberellins $A 4 / 7$ and cultural treatments: a review of the possible mechanisms. For Ecol Manage 19, 6584

Rohozinski J, Edwards GR, Hoskyns P (1986) Effects of brief exposure to nitrogenous compounds on floral initiation in apple trees. Physiol Veg 24, 673-677

Seiler N, Wiechman M (1970) TLC analysis of amines as their Dns-derivatives. In: Progress in thin layer chromatography and related methods (A Neiderwieser, G Pataki, eds) Humphrey Science, Ann Arbor, Vol 1, 95-144

Slocum RD, Kaur-Sawhney R, Galston AW (1984) The physiology and biochemistry of polyamines in plant. Arch Biochem Biophys 235(2), 283-303

Smith TA (1970) Putrescine, spermidine, and spermine in higher plants. Phytochemistry 9 , 1479-1486

Smith MA, Davies PJ (1985) Manipulation of the polyamine content and senescence of apical buds of G2 peas. Plant Growth Regul 3, 401417 
Smith MA, Davies PJ, Reid JB (1985) Role of polyamines in gibbereilin-induced internode growth in peas. Plant Physiol 78, 92-99

Smith MA, Davies PJ (1987) Monitoring polyamines in plant tissue by high performance liquid chromatography. In: High performance liquid chromatography in plant science (HF Linskens, JF Jachson, eds) 209-227

Steward FC, Durzan DJ (1965) Metabolism of organic nitrogenous compounds. In: Plant Physiology (FC Steward, ed) Academic Press, New York, 379-386
Suttle JC (1981) Effect of polyamines on ethylene production. Phytochemistry 20, 14771480

Tiburcio AF, Maséu MA, Dumortier FM, Galston AW (1986) Polyamine metabolism and osmotic stress. Plant Physiol 82, 347-369

Tiburcio AF, Kaur-Sawhney R, Galston AW (1988) Polyamine biosynthesis during vegetative and floral bud differentiation in thin layer tobacco tissue cultures. Plant Cell Physiol 29(7), 1241-1249 